LUNG CANCER

\section{OSNA enables more accurate detection of micrometastases}

The presence, or absence, of micrometastases in surgically removed lymph nodes has important implications for the prognosis of patients with non-small-cell lung cancer (NSCLC) following surgery, although standard histopathological methods do not enable the detection of all micrometastases. Now, new research indicates the superiority of the one-step nucleic acid amplification (OSNA) method over histopathological approaches in the detection of micrometastases.

A total of 885 lymph nodes were extracted during 64 different radical surgery procedures, with pooling of lymph nodes originating from the same compartment to create 320 distinct nodal zones. All lymph nodes were examined for the presence of micrometastases using three methods: haematoxylin and eosin staining, OSNA, and immunohistochemistry for cytokeratin 19 (an epithelial cell marker, expression of which is also detected using the OSNA assay).

Concordance between all three methods was reached in $89.1 \%$ of pooled samples, of which $82.2 \%$ were identified as negative for micrometastases and $6.9 \%$ positive by all three approaches. The OSNA method revealed the presence of micrometastases in $18 \%$ of samples, including $8.1 \%$ in which both other methods had failed to reveal the presence of micrometastases. Following further investigations, 13 patients in whom the OSNA method revealed lymph node micrometastases were reclassified as having NSCLC of a higher pTMN stage than originally diagnosed, with important implications for disease management.

These findings suggest that the OSNA method is superior to histopathological approaches for the detection of lymph node micrometastases following surgery. The outcomes of prospective evaluations of the validity of this approach are eagerly awaited.

\section{Peter Sidaway}

ORIGINAL ARTICLE Vodicka, J. et al. A more sensitive detection of micrometastases of NSCLC in lymph nodes using

the one-step nucleic acid amplification (OSNA) method.

J. Surg. Oncol. http://dx.doi.org/ 10.1002/jso.24826 (2017) 